\title{
Research on the Layout of General Aviation Emergency Rescue Spots Based on P-median Model
}

\author{
Na Lu', a , Liang Zhang, b, * \\ ${ }^{1}$ College of Economics and Management, Xi'an Aeronautical University, Xi'an710077, China \\ ${ }^{2}$ Airport Operation Management Department, Guangzhou Civil Aviation College Guangzhou 510403, China \\ a hr.luna@163.com, b25267764@qq.com \\ * corresponding author
}

Keywords: General aviation, rescue spot, Floyd algorithm, P-median algorithm

\begin{abstract}
This article is a research on the layout method of general aviation emergency rescue spots in city, and determines the site selection scheme based on p-median model and Floyd algorithm. The purpose is to minimize the maximum distance from the rescue spot to the disaster sites, and maximize the use of the flexibility, mobility, maximum efficiency and other functions of the general aviation emergency rescue. The presentation of this scheme is of great significance for the layout work of the general aviation emergency rescue spots.
\end{abstract}

\section{Introduction}

The opening of low-altitude airspace brings unprecedented opportunity to the development of general aviation. Especially on the capabilities of emergency rescue, the general aviation plays its irreplaceable role for other land and air transportation modes. With the economic and social development, the progress of urbanization is intensified, and the human activities are more frequent [1]. In recent years, many natural disasters and human disasters such as earthquakes, the explosion event in Tianjin, terrorist activities, flood etc. are all seen in China, and the emergency rescue plays an important role in these disasters. Air rescue is normally the general aviation rescue or military aircraft rescue. The general aviation rescue has its own unique superiority such as fast speed, highefficient, saving manpower and material resources, independence from complex terrain, and etc, which brings great convenience to the emergency rescue [2, 3].

\section{Method for the Layout of General Aviation Emergency Rescue Spots}

\subsection{Determination Method for the Number of Emergency Rescue Spots}

Suppose that the effective search and rescue range of general aviation aircraft for rescue presents a round shape, the area of its effective search and rescue range can be expressed as $s=\pi * r^{2}$. The formula (1) for the number of service areas which require deploying and controlling unmanned aerial vehicles is as below.

$$
N=\frac{s}{\pi * r^{2}}
$$

\subsection{Determination of the Deployment and Control Method for Emergency Rescue Spots}

All According to the model and algorithm research on the site selection of emergency system ${ }^{[5-7]}$, through the description on above problems, this article supposes the whole emergency network's site selection problem can be abstracted as an unoriented empowerment network $G=\{V, E\}$, wherein $V=\left\{V_{1}, V_{2}, \ldots, V_{n}\right\}$ is the set of each disaster site; $E=\left\{e_{1}, e_{2}, \ldots, e_{m}\right\}$ is the set of arcs between spots in the connection rescue network system, namely the air route between each disaster site. For 
any two spots in the network $G, i . e . X$ and $Y, d(X, Y)$ represents the shortest path between them. See formula (2) for the peculiarity of $d\left(V_{i}, X\right)$.

$$
\begin{gathered}
X \in\left(V_{p}, V_{q}\right) d\left(V_{i}, X\right)=\min \left\{d\left(V_{i}, V_{p}\right)+d\left(V_{p}, X\right), d\left(V_{i}, V_{q}\right)+d\left(V_{q}, X\right)\right\} \\
r_{i}=\left(d\left(V_{i}, V^{\prime}\right)+d\left(V_{i}, V^{\prime \prime}\right)\right) / 2 ; r=\min \left(r_{1}, r_{2}, r_{3}, \ldots, r_{n}\right)
\end{gathered}
$$

Fig 1 is the $v_{i}$-centered star network diagram which changes the symbol. Whereas, $v_{i}$ is the center apex; $V^{\prime}$ is the point farthest from the $v_{i}$ on the optimal path; $V^{\prime \prime}$ is the point having the farthest distance and not on the optimal path of $v_{i}, V^{\prime} ; V^{\prime \prime \prime}$ is the point having the farthest distance and not on the optimal path of $v_{i} 、 V^{\prime} ; v_{x}$ is the alternative emergency rescue spot.

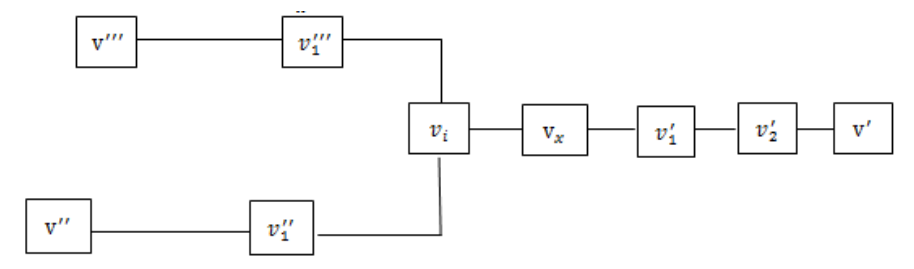

Fig 1 Star Network Diagram Centered by $v_{i}$

The calculation steps of the algorithm of emergency rescue spot location:(1)Find out the $V^{\prime}$ corresponding to the maximum value on row $i$ in the minimum distance matrix $S$; (2) Find out the $V^{\prime \prime}$ corresponding to the secondary maximum value in the matrix $S$;(3)According to the shortest path matrix $P$, judge whether $V^{\prime \prime}$ is on the optimal path from $v_{i}$ to $V^{\prime}$. If yes, turn back to Step (2), and continue to find out the secondary maximum value $V^{\prime \prime}$, and then move on to the next step; if no, turn to Step (4), and calculate the local radius;(4)Calculate the local radius of each row by Formula (1-3);(5)Calculate the absolute radius by Formula (1-4);(6)Determine the location of the absolute center, namely the emergency rescue spot.

\section{Analogue Simulation}

This part takes XX district of XX city as the target to analyze the feasibility of the deployment and control scheme for unmanned aerial vehicle rescue spots by using the model established for carrying out this research. Fig. 2 is the seven areas distributed on the map and some simple information, thus the number of apex points on the figure shall be 7 , and the number of rescue spots required shall be 3. It is concluded according to simple data process that, Figure 3-3 is the obtained figure for the distance between each area in the district and the traffic path through simplification (unit: km).

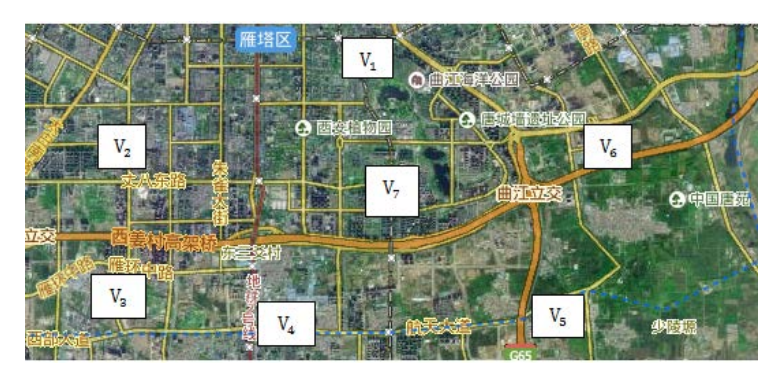

Fig 2 Geographic Distribution Map

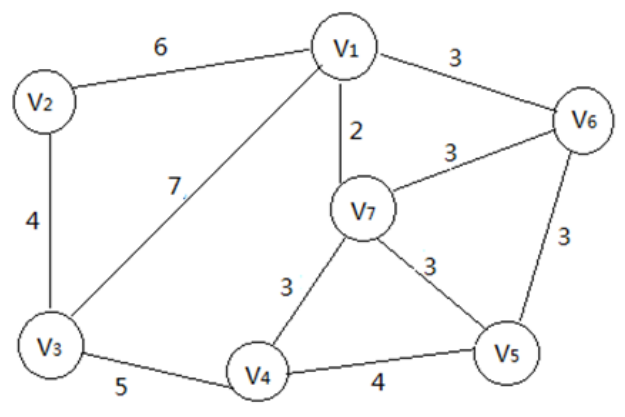

Fig 3 Simplified Distance Diagram

Firstly, the distance matrix and minimum distance matrix, and the shortest path matrix calculated by Floyd algorithm are as follows: 


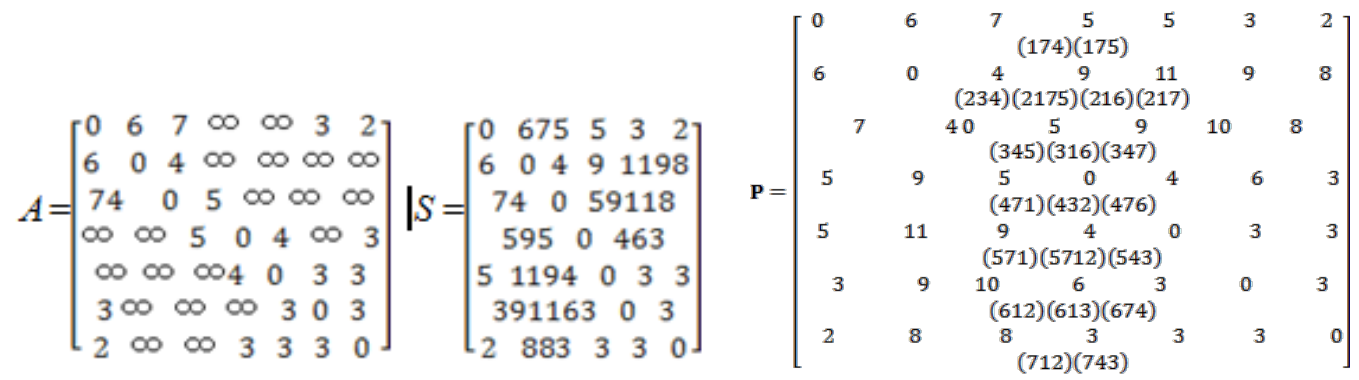

Secondly, the local center point and local radius rare obtained by Formula (3) through the minimum distance matrix $S$ and the shortest path matrix $P$.

$$
\begin{aligned}
& r_{1}=(7+6) / 2=6.5 ; r_{2}=(11+9) / 2=10 ; r_{3}=(11+9) / 2=10 \\
& r_{4}=(9+5) / 2=7 ; r_{5}=(11+9) / 2=10 ; r_{6}=(11+9) / 2=10 ; r_{7}=(8+3) / 2=5.5
\end{aligned}
$$

The absolute radius can be calculated as $r=\min \left(r_{1}, r_{2}, r_{3}, \ldots, r_{n}\right)=r_{7}=5.5$. So the absolute cen ter is on the connecting line 10-5.5=4.5 from $v_{7}$ to $v_{2}$, on the connecting line 10-5.5=4.5 from $v_{7}$ to $v_{3}$, on the connecting line $10-5.5=4.5$ from $v_{7}$ to $v_{5}$, and on the connecting line $10-5.5=4.5$ from $v_{7}$ to $v_{6}$.

In conclusion, the location of rescue spot $v_{x}$ can be selected as the three spots from the location of the four absolute centres above mentioned.

\section{Conclusion}

Through analyzing the influence factors of the layout scheme of general aviation emergency rescue spots, in consideration of the influence of each factor on the disaster site and the demands of establishing emergency rescue spot in surrounding areas aiming at the layout, combining the pmedian model and Floyd shortest path algorithm in the site selection problem for emergency facilities to minimize the maximum distance from the rescue spot to the disaster site so as to determine the number of rescue spots. This article raises the deployment and control scheme for emergency rescue spots for general aviation, which solves the layout problem of emergency rescue spots, to maximize the use of the flexibility, mobility, maximum efficiency and other functions of the general aviation emergency rescue. The presentation of this scheme is of great significance for the layout work of the general aviation emergency rescue spots.

\section{References}

[1]. Meng Jianjun. Give Play to the Effect of General Aviation in Emergency Rescue [J]. Disaster Reduction in China, 2011(11): 42.

[2]. Zhou Yujing \& The Yanbin. Overview of Current Situation on the Development of China's General Aviation [J]. China Civil Aviation, 2011 (12): 15.

[3]. [DB/OL]. http://www.sn.xinhuanet.com/2015-10/14/c_1116819265.htm, 2015-10-14.

[4]. Yang Zhengze. Current Situation and Countermeasures on the Development of China's General Aviation [J]. Macroeconomic Management, 2015 (11): 74.

[5]. Zhang Bing. Planning Ideas for General Aviation Emergency Rescue [J]. China Civil Aviation, 2008 [7], 20:20.

[6]. Zhuyan, Shao Quan, Jia Meng, Zhang Haijiao \& Zhang Jinshi. Research on the Layout Method of Emergency Rescue Spots for General Aviation [J]. Henan Science, 2015-02, 33 (02): 266.

[7]. Mou Qifeng \& Sun Xiren. Evaluation Methodology for Operation Security State of Airport [J]. Academic Journal of Traffic Engineering and Information, 2011, 9 (3): 12-16, 42. 
[8]. Wang Tai, Shi Qiong \& Jiang Cailiang. Layout Method for Helicopter Expressway Rescue Network Based on First-aid Time [J]. China Journal of Highway and Transport, 2011, 24 (03):100.

[9]. Huang Rongbing, Kim S,Menezes MBC. Facility Location for Large-scale Emergencies[J]. Annals of Operations Research, 2010, 181(1):271-286. 